\title{
Piecewise-linear Lyapunov functions for structural stability of biochemical networks
}

\author{
Franco Blanchini $^{1, \mathrm{a}}$, Giulia Giordano ${ }^{\mathrm{a}}$ \\ ${ }^{a}$ Dipartimento di Matematica e Informatica, Università degli Studi di Udine, Via delle Scienze 206, 33100 Udine, Italy
}

\begin{abstract}
We consider the problem of assessing structural stability of biochemical reaction networks with monotone reaction rates, namely of establishing if all the networks with a certain structure are stable regardless of specific parameter values. We investigate stability by absorbing the network equations in a linear differential inclusion and seeking for a polyhedral Lyapunov function proper to the considered network structure. A numerical recursive procedure is devised to test stability. For a wide class of mono- and bimolecular reaction networks, which we name unitary, the procedure is shown to be very efficient since, due to the particular structure of the problem, it requires iterations in the space of integer-valued matrices. We also consider a similar, less conservative procedure that allows us to test, even when the Lyapunov function cannot be found, whether the system evolution is structurally bounded. In this case, we absorb the equations in a positive linear differential inclusion. To show the effectiveness of the proposed procedure, we report the outcomes of both a stability and a boundedness test, for many non-trivial biochemical reaction networks, and we analyze well established models in the literature.
\end{abstract}

Key words: biochemical networks; biochemical systems; structural stability; global stability; piecewise-linear Lyapunov functions; graph

\section{Introduction}

A vast literature agrees on the fact that chemical and biochemical networks suffer of a major trouble: their parameters are widely uncertain, time varying and depending on unpredictable factors due to specific working conditions. On the other hand, it is also recognized that particular behaviors depend on particular structures, regardless of specific parameter values. Structural investigation aims at explaining how and why certain systems perform the proper tasks in completely different conditions [2].

If all the systems of a class characterized by a structure have a certain property regardless of parameter values, such a property is called structural (see for instance [31], [9], [22]). This concept is deeply related with robustness [13], [18], with the difference that the latter concept is usually attributed to systems which can work under large parameter variations.

Structural analysis of chemical reaction networks, begun in the early Seventies [26], [24], [25], has provided fundamental results. Among the most celebrated are the zerodeficiency theorem and the one-deficiency theorem [19], [20], [21]. The zero-deficiency theorem provides a structural general sufficient condition (0-deficiency) assuring that a

\footnotetext{
1 Corresponding author. Email: blanchini@uniud.it
}

chemical network described by mass action kinetics admits a single positive stable equilibrium; 0 -deficiency is immediately verifiable from an easy test on the network structure (i.e. the reactions) and the proof nicely adopts the system entropy as a Lyapunov function. These results are still attracting a lot of attention [14, 15], [11], [3], [23]. One fundamental assumption in the zero-deficiency theorem requires the reaction kinetics to be of the mass action type, hence polynomial (although a possible generalization is proposed in [33]). This is a widely accepted assumption; still there are cases in which it is not necessarily satisfied, for instance non-perfectly mixed systems.

In this paper we investigate stability without the mass action kinetics assumption: we only require monotonicity of reaction rates. We make use of polyhedral Lyapunov functions, which have been successfully employed in the robustness analysis of uncertain systems (see [8] for a literature survey) and have been used to prove the stability of compartmental systems [29]. Compartmental systems are special cases of monotone systems [32] and can be thought as monomolecular chemical reactions in which each species can be transformed into another (e.g. $A \stackrel{g(a)}{\longrightarrow} B)$. Under the assumption of increasing reaction rate, stability can be proved by adopting as a Lyapunov function the 1-norm, which is a particular polyhedral (or piecewise-linear) norm.

Recent attempts in using polyhedral norms as candidate Lya- 
punov functions for biochemical networks have been proposed in [9], [22], although applied to quite specific problems.

The main idea of this paper is to investigate structural stability of a wide category of chemical reaction networks by adopting as candidate Lyapunov functions polyhedral norms, including the 1-norm as a special case. The main result is a procedure to generate piecewise-linear Lyapunov functions which may certify the stability of all chemical reaction networks with a certain structure. To have an intuition of how a structure looks like, we suggest the reader to give a preliminary look at Fig. 4, where several possible cases are depicted. If a piecewise-linear Lyapunov function is derived, network stability is structural, in the sense that, under some general monotonicity assumptions, it is assured for all reaction rate functions. Consider, for example, the network corresponding to the graph named Brahms5 in Fig. 4. The degradation reaction $A+E \rightarrow \emptyset$ introduces a negative feedback from the final product $E$ to $A$, which could be potentially destabilizing. Yet, by finding a suitable polyhedral Lyapunov function, we can demonstrate that the system is structurally stable, for any choice of the reaction rate functions.

The contributions of the paper can be summarized as follows.

- We consider general chemical networks, both isolated and with external inputs, under general monotonicity assumptions on the involved reaction rate functions, thus without restricting to mass action kinetics reactions.

- Based on the network structure only, we seek a polyhedral Lyapunov function (actually a norm) for the system, by absorbing the nonlinear system in a linear differential inclusion.

- We show that the existence of a polyhedral Lyapunov function is equivalent to the stability of a proper discrete difference inclusion.

- A recursive procedure, based on the discrete difference inclusion, is employed to generate the unit ball of the polyhedral norm. In the case of unitary reaction networks, in which the stoichiometric matrix has coefficients in $\{-1,0,1\}$, the procedure enormously benefits from the fact that iterations occur in the set of integer-valued matrices.

- The results in [29] follow as a special case, since the procedure generates the 1-norm for compartmental systems.

- We show that a similar procedure can be adopted, when structural stability is not satisfied, to prove at least boundedness of the state variables.

- We show that, once a polyhedral Lyapunov function is found, we can investigate local stability of the equilibrium in isolated systems within the stoichiometric compatibility class.

- We investigate structural stability of an extensive set of networks by our method. Surprisingly enough, non-trivial systems can be managed without difficulties, providing either a positive certificate (by finding a piecewise linear function with quite a small number of vertices) or a negative certificate (non-existence of such a function).

\section{Structural stability analysis}

\subsection{Model description and assumptions}

We denote chemical species with uppercase letters and their concentrations with the corresponding lowercase letter. We consider the class of models

$$
\dot{x}=S g(x)+g_{0}
$$

where the state $x \in \mathbb{R}_{+}^{n}$ represents the concentration of biochemical species, $g(x) \in \mathbb{R}^{m}$ is a vector of functions representing the reaction rates and $g_{0} \geq 0$ is a vector of constant influxes; $S \in \mathbb{Z}^{n \times m}$ is the stoichiometric matrix of the system, whose entries $s_{i j}$ represent the net amount of the $i$-th species produced or consumed by the $j$-th reaction, excluding the contribution of constant influxes.

Assumption 1 All the component functions of vector $g(x)$ are nonnegative and continuously differentiable. All their partial derivatives are positive in the positive orthant.

Decreasing trends can be considered as well: in some cases, this just requires changing sign to $g$. An important case is that of a species which is present in a total amount $\bar{x}_{i}>0$ and can be either active, $x_{i}$, or inactive, $x_{i}^{*}$, with $x_{i}+x_{i}^{*}=\bar{x}_{i}$. Since $0 \leq x_{i} \leq \bar{x}_{i}$, the activation term must be the only positive term in the right side of the equation. For instance, the equation

$$
\dot{a}=-g_{i n}(a, b)+g_{a c t}(\bar{a}-a, c)
$$

includes the inhibition term $g_{\text {in }}$ and the activation term $g_{a c t}$.

Assumption 2 Each component function of vector $g(x)$ is zero if and only if at least one of its arguments is zero. Moreover, if $s_{i j}<0$, then $g_{j}$ must depend on $x_{i}$.

Assumption 2, ensuring that for $x_{i}=0$ we have $\dot{x}_{i} \geq 0$, is required to guarantee that (1) is a positive system. For instance, $g_{\text {in }}(a, b)$ in (2) can be of the form $\kappa \frac{b a}{1+a}$, but not $\kappa \frac{b}{1+a}$.

Example 2.1 The chemical reactions [7, 27]

$$
\begin{array}{ll}
A+B \stackrel{g_{a b}(a, b)}{A^{*},} & B^{*} \stackrel{g_{b}\left(b^{*}\right)}{\longrightarrow} B, \\
C+A^{*} \stackrel{g_{a c}\left(a^{*}, c\right)}{ } A+B^{*}, & \emptyset \stackrel{c_{0}}{\longrightarrow} C
\end{array}
$$

involve the genelet species $A$ (and its inactive form $A^{*}$ ), the inhibitor strand $B$ (and its inactive form $B^{*}$ ) and the $R N A$ output $C$. Along with the mass conservation constraints $\bar{a}=a+a^{*}$ and $\bar{b}=b+b^{*}+a^{*}$, these reactions correspond to the following ODEs for $x=\left[\begin{array}{lll}a & b & c\end{array}\right]^{\top}$ :

$$
\begin{aligned}
\dot{a} & =g_{a c}(\bar{a}-a, c)-g_{a b}(a, b) \\
\dot{b} & =g_{b}(\bar{b}-\bar{a}+a-b)-g_{a b}(a, b) \\
\dot{c} & =c_{0}-g_{a c}(\bar{a}-a, c)
\end{aligned}
$$


In this case we have

$$
\begin{gathered}
S=\left[\begin{array}{rrr}
1 & -1 & 0 \\
0 & -1 & 1 \\
-1 & 0 & 0
\end{array}\right], g(x)=\left[\begin{array}{c}
g_{a c}(\bar{a}-a, c) \\
g_{a b}(a, b) \\
g_{b}(\bar{b}-\bar{a}+a-b)
\end{array}\right], \\
g_{0}=\left[\begin{array}{lll}
0 & 0 & c_{0}
\end{array}\right]^{\top} .
\end{gathered}
$$

A (non-exhaustive) list of possible reactions, together with the corresponding reaction terms appearing in the proper equations, is reported next.

\section{List of possible reactions}

(a) $\emptyset \stackrel{a_{0}}{\longrightarrow} A: \quad \dot{a}=a_{0}, \quad a_{0}$ constant

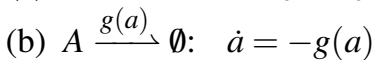

(c) $A \stackrel{g(a)}{\longrightarrow} B: \quad \dot{a}=-g(a), \dot{b}=g(a)$

(d) $A+B \stackrel{g(a, b)}{\emptyset:} \quad \dot{a}=-g(a, b), \dot{b}=-g(a, b)$

(e) $A+B \stackrel{g(a, b)}{\longrightarrow} C$ :

$$
\dot{a}=-g(a, b), \dot{b}=-g(a, b), \dot{c}=g(a, b)
$$

(f) $A \stackrel{g(a)}{\longrightarrow} B+C: \quad \dot{a}=-g(a), \dot{b}=g(a), \dot{c}=g(a)$

(g) $A+B \stackrel{g(a, b)}{\longrightarrow} C+D$ :

$$
\dot{a}=-g(a, b), \dot{b}=-g(a, b), \dot{c}=g(a, b), \dot{d}=g(a, b)
$$

(h) Activation. $A^{*}+B \stackrel{g\left(a^{*}, b\right)}{\longrightarrow} A$ :

$$
\dot{a}=g(\bar{a}-a, b), \dot{b}=-g(\bar{a}-a, b)
$$

with $a+a^{*}=\bar{a}$, where $\bar{a}$ is the total concentration

(i) Difference dependence (see Example 2.1).

$$
\dot{a}=g(\bar{a}-\bar{b}-a+b)
$$

Any network can be represented by a graph, whose nodes are associated with biochemical species, while the arcs represent interactions. In Fig. 1 we define the arcs corresponding to the reactions listed above. The graph of Example 2.1 corresponds to that named Albinoni3 in Fig. 4.

Assumption 3 Functions $g_{j}(\cdot)$ in which each argument depends on a single variable $x_{i}$ are admitted if $s_{i j} \frac{\partial g_{j}}{\partial x_{i}}<0$ for each argument. Functions having as an argument the sum or difference of more variables, such as $g_{j}\left( \pm x_{i} \pm x_{k}\right)$, are admitted if they appear in a single equation, $\dot{x}_{k}=\ldots$, and $s_{k j} \frac{\partial g_{j}}{\partial x_{k}}<0$.

Note that the diagonal entries of the Jacobian of $S g(x)$ are negative and no autocatalytic reactions are considered. Also

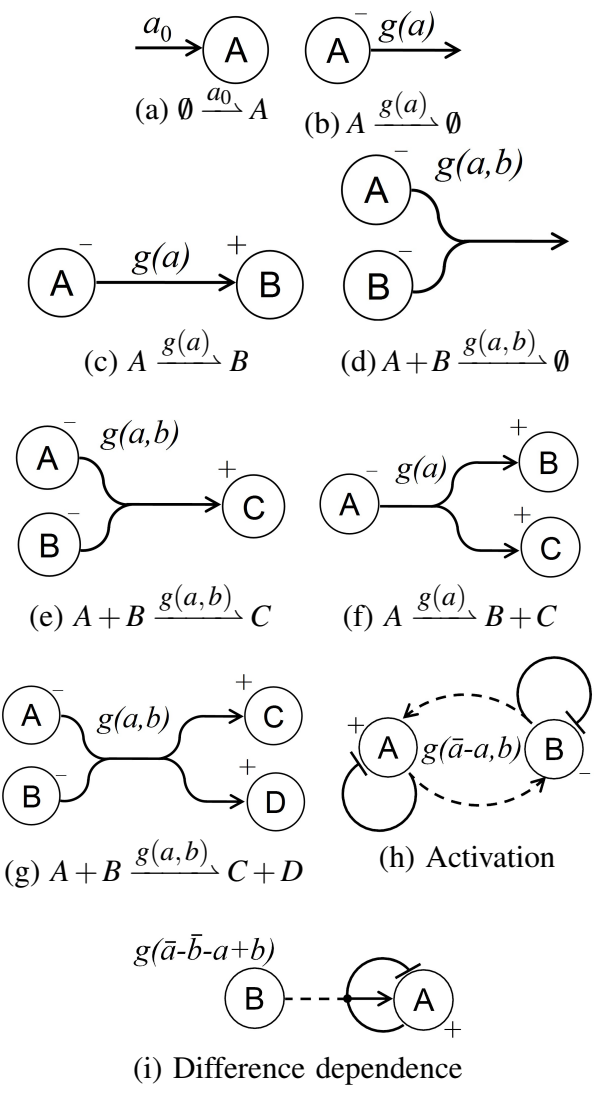

Fig. 1. Graph representations of biochemical reactions.

reactions of the form $A \rightarrow A+B$, often used to model gene expression, are not allowed. Nevertheless, we successfully analyze a more complete gene expression model later.

Networks composed of reactions in the list (Fig. 1) are a subset of those satisfying Assumption 3. These networks are also unitary, according to the next definition.

Definition 2.1 The network is unitary if $s_{i j} \in\{-1,0,1\}$.

Remark 2.1 Requiring a network to be unitary is a restriction: for instance, multimolecular reactions of the type $n A+B \rightarrow P$, with $n>1$, would be ruled out. We notice however two basic points. 1) With regard to our setup, the restriction is not theoretical, but essentially computational. In fact, our theory works in general, although the numerical procedure might not converge for non-unitary networks. We will reconsider this issue in Section 4. 2) Multimolecular reactions can be always expressed as a cascade of bimolecular reactions, which are unitary. For instance, $2 A+B \rightarrow P$ can be decomposed as the pair of unitary reactions $A+B \rightarrow C$ and $C+A \rightarrow P$. Such a decomposition is justified, since trimolecular reactions are considered unlikely to happen and no reactions concurrently involving more than three molecules have yet been observed; therefore an overall reaction is more plausibly modeled by a chain of bimolecular steps (see, for instance, [17] Section 7.4 or [28] Sections 2.1, 2.3). 
In the paper we consider both isolated systems $\left(g_{0}=0\right)$ and non-isolated systems $\left(g_{0} \neq 0\right)$. We remind that for $g_{0}=0$ the solution of the system is forced to stay in the stoichiometric compatibility class $\mathscr{C}(x(0))$ :

$$
x(t) \in \mathscr{C}(x(0))=\{x(0)+\operatorname{Ra}[S]\} \cap \mathbb{R}_{+}^{n} .
$$

Example 2.2 Consider the reactions

$$
X_{1} \underset{g_{2}\left(x_{2}\right)}{\stackrel{g_{1}\left(x_{1}\right)}{\rightleftharpoons}} X_{2}, \quad \emptyset \stackrel{g_{01}}{\longrightarrow} X_{1}, \quad \emptyset \stackrel{g_{02}}{\longrightarrow} X_{2}
$$

corresponding to the second order linear system

$$
\left[\begin{array}{c}
\dot{x}_{1} \\
\dot{x}_{2}
\end{array}\right]=\left[\begin{array}{cc}
-1 & 1 \\
1 & -1
\end{array}\right]\left[\begin{array}{l}
g_{1}\left(x_{1}\right) \\
g_{2}\left(x_{2}\right)
\end{array}\right]+\left[\begin{array}{l}
g_{01} \\
g_{02}
\end{array}\right]
$$

Then, for $g_{0}=0$, the stoichiometric compatibility class is

$$
\mathscr{C}(x(0))=\left\{x_{1}+x_{2}=x_{1}(0)+x_{2}(0), \quad x_{1}, x_{2} \geq 0\right\} .
$$

In view of our structural analysis, we introduce the following definition of stability. Consider the $\varepsilon$-modified system

$$
\dot{x}(t)=-\varepsilon x(t)+S g(x(t))+g_{0}
$$

with $\varepsilon>0$ arbitrarily small.

Definition 2.2 System (1) is

i) structurally stable if any equilibrium point $\bar{x}$ of the system with $g_{0}=0$ is Lyapunov stable: there exists a continuous, strictly increasing and unbounded function $\omega: \mathbb{R}_{+} \rightarrow \mathbb{R}_{+}$, with $\omega(0)=0$, such that $\|x(t)-\bar{x}\| \leq \omega(\|x(0)-\bar{x}\|)$;

ii) structurally convergent if it is structurally stable and, for any $\varepsilon>0$ and $g_{0} \geq 0$, the perturbed system (3) has globally bounded solutions and admits an equilibrium which is globally asymptotically stable in $\mathbb{R}_{+}^{n}$.

The infinitesimal parameter $\varepsilon>0$ in (3) is required for technical reasons. We consider parameters whose values are sign definite but unknown, thus can be arbitrarily close to zero. Therefore, after absorbing the system in a differential inclusion, we are not able to asses in general asymptotic stability without considering a natural degradation of the species, represented by $\varepsilon>0$. The introduction of $\varepsilon$ in Definition 2.2 ii) does not lead to the classification as stable of systems which are unstable. Moreover, such a degradation term is necessary for the system to tolerate persistent positive inputs. For instance, according to our definition, the system of Example 2.2 is structurally stable and convergent. Without any $\varepsilon$-degradation, it would produce unbounded solutions unless $g_{0}=0$. For $g_{0}=0$ any of its equilibrium points is only marginally stable. However, it has the property of asymptotic stability within the stoichiometric compatibility class, a problem we will treat later, without considering any $\varepsilon>0$.

\subsection{Absorbing the system in a differential inclusion}

For sake of simplicity in the exposition, we start by assuming that an equilibrium $\bar{x}=\bar{x}(\varepsilon)$ exists $\forall \varepsilon>0$ and we give a criterion for global stability. We will see later that, if the system passes the computational test we propose, such an equilibrium indeed exists.

Let $z \doteq x-\bar{x}$, thus $x=z+\bar{x}$. Since $0=S g(\bar{x})-\varepsilon \bar{x}+g_{0}$, we can write

$$
\dot{z}(t)=S[g(z(t)+\bar{x})-g(\bar{x})]-\varepsilon z(t) .
$$

Then we have the following.

Proposition 2.1 System (4) can be equivalently written as

$$
\dot{z}(t)=B D(z(t)) C z(t)-\varepsilon z(t)
$$

where matrix $B \in \mathbb{Z}^{n \times q}$ is formed by a selection of columns of $S, C \in \mathbb{Z}^{q \times n}$ and $D(z)$ is a diagonal matrix with nonnegative diagonal entries. $q$ is the number of possible partial derivatives with respect to all arguments $(q \geq n, q \geq m)$.

Proof To show that (4) can be equivalently written as the differential inclusion (5), consider the generic function $g_{k}\left(x_{i}, x_{j}\right)$ (the argument applies to functions of more than two variables) and write it as

$$
\begin{aligned}
& g_{k}\left(z_{i}+\bar{x}_{i}, z_{j}+\bar{x}_{j}\right)-g_{k}\left(\bar{x}_{i}, \bar{x}_{j}\right)= \\
& \frac{g_{k}\left(z_{i}+\bar{x}_{i}, z_{j}+\bar{x}_{j}\right)-g_{k}\left(\bar{x}_{i}, z_{j}+\bar{x}_{j}\right)}{z_{i}} z_{i} \\
& +\frac{g_{k}\left(\bar{x}_{i}, z_{j}+\bar{x}_{j}\right)-g_{k}\left(\bar{x}_{i}, \bar{x}_{j}\right)}{z_{j}} z_{j}= \\
& \frac{\partial g_{k}\left(\tilde{x}_{i}, \bar{x}_{j}\right)}{\partial x_{i}} z_{i}+\frac{\partial g_{k}\left(\bar{x}_{i}, \tilde{x}_{j}\right)}{\partial x_{j}} z_{j}=\left( \pm D_{k i}\right) z_{i}+\left( \pm D_{k j}\right) z_{j},
\end{aligned}
$$

in view of the differential mean value theorem, where $\tilde{x}_{i}$ and $\tilde{x}_{j}$ are proper values in the intervals $\left[\bar{x}_{i}, z_{i}+\bar{x}_{i}\right]$ and $\left[\bar{x}_{j}, z_{j}+\right.$ $\left.\bar{x}_{j}\right]$ respectively, while $D_{k i}$ and $D_{k j}$ are nonnegative scalar functions of $\bar{x}$ and $z$. Note that the values $\tilde{x}_{i}$ and $\tilde{x}_{j}$ are not necessarily unique and depend on the function $g_{k}$. Let us order the partial derivatives $D_{k i}$ and $D_{k j}$ as $D_{1}, D_{2}$, etc. Then $D=\operatorname{diag}\left\{D_{1}, D_{2}, \ldots, D_{q}\right\}$. Matrix $B$ is achieved by replicating (up to the sign) each column of $S$, say the $k$-th, a number of times equal to the arguments of $g_{k}$. In matrix $C, c_{k i}$ is \pm 1 if $g_{k}$ depends on $x_{i}, 0$ if it does not.

Example 2.3 Consider the system of Example 2.1 and let $D_{1}=-\frac{\partial g_{a c}}{\partial a}, D_{2}=\frac{\partial g_{a b}}{\partial a}, D_{3}=\frac{\partial g_{a b}}{\partial b}, D_{4}=\frac{\partial g_{a c}}{\partial c}, D_{5}=\frac{\partial g_{b}}{\partial a} b e$ positive parameters. Then $D=\operatorname{diag}\left(D_{1}, D_{2}, D_{3}, D_{4}, D_{5}\right)$,

$B=\left[\begin{array}{ccccc}-1 & -1 & -1 & 1 & 0 \\ 0 & -1 & -1 & 0 & 1 \\ 1 & 0 & 0 & -1 & 0\end{array}\right], \quad C=\left[\begin{array}{ccccc}1 & 1 & 0 & 0 & 1 \\ 0 & 0 & 1 & 0 & -1 \\ 0 & 0 & 0 & 1 & 0\end{array}\right]^{\top}$ 
Remark 2.2 Denoting by $b_{i}$ the $i$-th column of matrix $B$ and by $c_{i}^{\top}$ the $i-t h$ row of matrix $C$, Assumption 3 implies that

$$
c_{i}^{\top} b_{i}<0 \quad \forall i
$$

For unitary networks we have $c_{i}^{\top} b_{i}=-1 \forall i$, with noteworthy numerical benefits.

We have the following theorem.

Theorem 2.1 Consider the linear differential inclusion

$$
\dot{x}(t)=\left[-\varepsilon I+\sum_{i=1}^{q} b_{i} d_{i}(t) c_{i}^{\top}\right] x(t), \quad x(0)=x_{0}
$$

where $d_{i}(t)$ are arbitrary nonnegative scalar piecewise continuous functions ${ }^{2}$. Then:

1. stability of (7) for $\varepsilon=0$ implies structural stability of any equilibrium of (1);

2. asymptotic stability of (7) for $\varepsilon>0$ implies structural convergence of (3).

Proof Part 1 . is standard. If $\bar{x}$ is any equilibrium point, then, denoting by $z=x-\bar{x}$, we can absorb the nonlinear system in the linear differential inclusion, in the sense that all the solutions $z(t)$ are also solutions of (7) for $\varepsilon=0$. Then stability of (7) implies structural stability.

For part 2., the proof would be exactly the same if we could assume that (3) admits a steady state $\bar{x}$ for any $\varepsilon>0$. Indeed, once again, the set of solutions of the differential inclusion (5) is a subset of the set of solutions of (7). Therefore, we just have to prove that, if (7) is asymptotically stable, then (3) admits a steady state. Its global stability is then immediate.

Let $\tilde{x}$ be an arbitrary constant vector and define $z=x-\tilde{x}$. Write (3) as

$$
\begin{aligned}
\dot{z} & =\dot{x}=-\varepsilon x+S g(z+\tilde{x})+g_{0} \\
& =-\varepsilon z+S[g(z+\tilde{x})-g(\tilde{x})]+\tilde{g}_{0}=-\varepsilon z+B D(z, \tilde{x}) C+\tilde{g}_{0},
\end{aligned}
$$

where $\tilde{g}_{0} \doteq g_{0}+S g(\tilde{x})-\varepsilon \tilde{x}$ is a constant vector and $D$ a nonnegative diagonal matrix. Then the system can be written as (7) with an additive constant term, as follows:

$$
\dot{z}(t)=[-\varepsilon I+B D(t) C] z(t)+\tilde{g}_{0} .
$$

If (7) is asymptotically stable, then the solutions of this system are bounded. Since they are a superset of the solutions of (3), the latter has bounded solutions. Then it necessarily admits a steady state value $\bar{x}$ (depending on $\varepsilon$ ).

\footnotetext{
2 we mean that the number of discontinuity points is finite in each finite interval and, in each of these intervals, right and left limits are finite
}
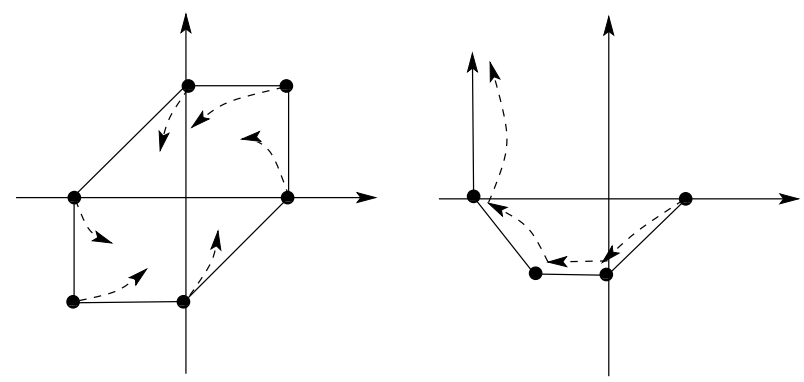

Fig. 2. The idea: convergent case (left); divergent case (right).

Remark 2.3 The stability of the differential inclusion is a sufficient condition for the stability of the original system. As we will see later, a polyhedral function exists for the former iff it exists for the latter.

From the proof of Theorem 2.1 the next Corollary follows.

Corollary 2.1 If the differential inclusion (7) is asymptotically stable, then (3) admits an equilibrium.

We conclude the section by pointing out the following equivalence.

Proposition 2.2 Stability of (7) for $\varepsilon=0$ is equivalent to its asymptotic stability for $\varepsilon>0$.

Proof It follows immediately by noting that, given $d_{i}(t) \geq 0$ and denoting by $x_{0}(t)$ the solution corresponding to $\varepsilon=0$, the solution corresponding to $\varepsilon>0$ is

$$
x_{\varepsilon}(t)=e^{-\varepsilon t} x_{0}(t) .
$$

\section{Analysis of the differential inclusion}

The main idea is depicted in Fig. 2. We associate the differential inclusion with a "nice" discrete-time difference inclusion. Then we prove that, if all the possible discrete transitions starting from the vertices of the diamond (the unit ball of $\|x\|_{1}$ ) remain bounded, then the continuous-time solution remains trapped inside the convex hull of the reached points (stable case). Conversely, if the difference inclusion diverges, so does the differential inclusion, since we prove that there exist continuous-time solutions arbitrarily close to the discrete-time solutions.

Let us start by considering the case $\varepsilon=0$ in (7). Since $d_{i}(t)$ are arbitrary nonnegative, the best we can do is to prove marginal stability ${ }^{3}$. For any state value $x \in \mathbb{R}^{n}$, the set of

\footnotetext{
3 for instance, $\dot{x}(t)=-d(t) x(t), d(t)>0$ is not necessarily asymptotically stable: the solution does not converge to 0 if $d(t)$ goes to 0 too quickly
} 
all derivatives is included in a cone of directions $\pm b_{i}$ :

$$
\dot{x} \in\left\{v: v=\sum_{i=1}^{q} v_{i} d_{i}, \quad d_{i} \geq 0\right\}, \quad \text { where } v_{i}=b_{i} c_{i}^{\top} x .
$$

Therefore, if we are able to show the existence of a common convex Lyapunov function for all the special systems

$$
\dot{x}=b_{i} c_{i}^{\top} x
$$

with $i=1, \ldots, q$, then the same function is a Lyapunov function for the differential inclusion (and the nonlinear system).

The solutions of (8) with initial state $x_{0}$ can be written as

$$
x(t)=x_{0}+\int_{0}^{t} b_{i} c_{i}^{\top} x(\sigma) d \sigma=x_{0}+b_{i} \vartheta(t)
$$

where $\vartheta(t)=\int_{0}^{t} c_{i}^{\top} x(\sigma) d \sigma$. To find $\vartheta$ we consider the variable $\left(c_{i}^{\top} x\right)$, which satisfies the differential equation

$$
\frac{d}{d t}\left(c_{i}^{\top} x\right)=\left(c_{i}^{\top} b_{i}\right)\left(c_{i}^{\top} x\right)
$$

Its solution is $c_{i}^{\top} x(t)=c_{i}^{\top} x_{0} e^{c_{i}^{\top} b_{i} t}$ and asymptotically converges to zero, since $c_{i}^{\top} b_{i}<0$ (see Remark 2.2). Then $x(t)$ converges to a finite value $x_{\infty}$. Without computing the integral, we achieve the limit as the value for which

$$
c_{i}^{\top} x_{\infty}=c_{i}^{\top}\left(x_{0}+b_{i} \vartheta_{\infty}\right)=0
$$

which yields $\vartheta_{\infty}=-c_{i}^{\top} x_{0} /\left(c_{i}^{\top} b_{i}\right)$.

The dynamics (8) asymptotically drives the state from $x_{0}$ to

$$
x_{\infty}=x_{0}+b_{i} \vartheta_{\infty}=x_{0}-\frac{b_{i} c_{i}^{\top} x_{0}}{c_{i}^{\top} b_{i}}=\left[I-\frac{b_{i} c_{i}^{\top}}{c_{i}^{\top} b_{i}}\right] x_{0} .
$$

Now let us consider the family of matrices

$$
\mathscr{F}=\left\{\Phi_{i} \doteq\left[I-\frac{b_{i} c_{i}^{\top}}{c_{i}^{\top} b_{i}}\right], \quad i=1, \ldots q\right\}
$$

and the discrete linear difference inclusion (actually a discrete-time switching system) $y_{k+1}=\Phi(k) y_{k}$, where $\Phi(k)$ is an arbitrary sequence in $\mathscr{F}$ and $y_{k} \in \mathbb{R}^{n}$. We can show that discrete and continuous-time stability are equivalent.

Theorem 3.1 Robust stability of

$$
\dot{x}(t)=B D(t) C x(t), \quad d_{i}(t) \geq 0
$$

is equivalent to robust stability of

$$
y_{k+1}=\Phi(k) y_{k}, \quad \Phi(k) \in \mathscr{F} .
$$

To prove this theorem, we use a technical lemma which states that any solution of the discrete-time system is approached by a solution of the continuous-time system for large enough time.

Lemma 3.1 Given systems (10) and (11), both starting from the initial condition $y_{0}=x(0)$, then $\forall k>0$ and $\forall \delta>0$, no matter how small, there exists $t>0$ such that $\left\|x(t)-y_{k}\right\|<\delta$.

Proof Denote by $D_{i}$ a matrix which has a 1 in the $i$-th diagonal position and 0 elsewhere. Take any sequence $y_{1}, y_{2}, \ldots$, $y_{k}$ solution of (11). At the first step we have $y_{1}=\Phi(0) y_{0}$, with $\Phi(0)=I-b_{i_{0}} c_{i_{0}}^{\top} /\left(c_{i_{0}}^{\top} b_{i_{0}}\right)$. Choose $D(t)=D_{i_{0}}$. By keeping such a $D(t)$ on a sufficiently large interval $\left[0, t_{1}\right)$, since the solution of system (10) converges to $y_{1}=\Phi(0) y_{0}$, there exists $t_{1}>0$ large enough such that, no matter how small $\delta_{1}>0$ is taken, $\left\|x\left(t_{1}\right)-y_{1}\right\|<\delta_{1}$. Now consider the second step $y_{2}=\Phi(1) y_{1}, \Phi(1)=I-b_{i_{1}} c_{i_{1}}^{\top} /\left(c_{i_{1}}^{\top} b_{i_{1}}\right)$, for some $i_{1}$, and take $D(t)=D_{i_{1}}$ on an interval $\left[t_{1}, t_{2}\right)$ to reach a state $x\left(t_{2}\right)$. Similarly we have that, for $t_{2}$ large enough, no matter how small $\delta_{2}>0$ is taken,

$$
\left\|x\left(t_{2}\right)-\Phi(1) x\left(t_{1}\right)\right\|<\delta_{2}
$$

To estimate the distance of $x\left(t_{2}\right)$ from $y_{2}=\Phi(1) y_{1}$, note that

$$
\begin{aligned}
\left\|x\left(t_{2}\right)-y_{2}\right\| & \leq\left\|x\left(t_{2}\right)-\Phi(1) x\left(t_{1}\right)\right\|+\left\|\Phi(1)\left(x\left(t_{1}\right)-y_{1}\right)\right\| \\
& \leq \delta_{2}+\|\Phi(1)\| \delta_{1}
\end{aligned}
$$

Then $\left\|x\left(t_{3}\right)-y_{3}\right\| \leq \delta_{3}+\|\Phi(2)\| \delta_{2}+\|\Phi(2)\|\|\Phi(1)\| \delta_{1}$ can be proved in the same way, by applying the proper $D(t)=D_{i_{2}}$ on a sufficiently large interval $\left[t_{2}, t_{3}\right)$, and so recursively:

$$
\begin{aligned}
& \left\|x\left(t_{k}\right)-y_{k}\right\| \leq \delta_{k}+\|\Phi(k-1)\| \delta_{k-1}+\|\Phi(k-1)\|\|\Phi(k-2)\| \delta_{k-2} \\
& +\cdots+\|\Phi(k-1)\| \ldots\|\Phi(2)\|\|\Phi(1)\| \delta_{1} .
\end{aligned}
$$

Since $\delta_{k}$ are arbitrarily small and $\|\Phi(k)\|$ are uniformly bounded, we can conclude $\left\|x(t)-y_{k}\right\|<\delta$ for some $t>0$.

Lemma 3.1 is interesting per se, because it demonstrates that the stability of the continuous-time system implies that of the discrete-time system. This depends on the peculiar properties of the systems we consider, since in general only the opposite is true.

Proof of Theorem 3.1 If the continuous-time system (10) is stable, for [6], Theorem 5.2, there exists a convex and compact set including 0 in its interior $(C$-set) which is robustly positively invariant for (10). Let us call such a set $\mathscr{S}$. We show that $\mathscr{S}$ must be robustly positively invariant also for (11). By contradiction, assume that for $y_{0} \in \partial \mathscr{S}$ (the boundary of $\mathscr{S}$ ) we have

$$
y_{1}=\Phi_{i} y_{0} \notin \mathscr{S} \quad \text { for some } \Phi_{i} \in \mathscr{F} .
$$

Then there exists a neighborhood $\mathscr{B}$ of $y_{1}$ such that $\mathscr{B} \cap \mathscr{S}=\emptyset$. In view of Lemma 3.1 we would have a 
continuous-time solution $x(t)$ approaching $y_{1}$ arbitrarily closely, hence entering in $\mathscr{B}$ and thus violating the assumption that $\mathscr{S}$ is positively invariant for (10).

Now assume that the discrete-time system (11) is stable. Then there exists a $C$-set $\mathscr{S}$ which is positively invariant for (11) ([6], Theorem 5.1). Consider $y_{0} \in \partial \mathscr{S}$. Then, by the definition of $\Phi_{i}$,

$$
y_{1}=\Phi_{i} y_{0}=\left[I-\frac{b_{i} c_{i}^{\top}}{c_{i}^{\top} b_{i}}\right] y_{0} \in \mathscr{S} .
$$

Define the Bouligand tangent cone to $\mathscr{S}$ in $y_{0}$ (see Fig. 3) as

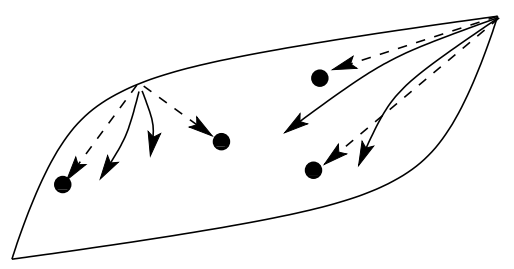

Fig. 3. The continuous-time solutions point inside the cone defined by the discrete-time solutions (dashed arrows)

$$
\mathscr{T}\left(y_{0}\right)=\left\{z: \lim _{h \rightarrow 0} \frac{\operatorname{dist}\left(y_{0}+h z, \mathscr{S}\right)}{h}=0\right\},
$$

where $\operatorname{dist}(u, \mathscr{S})$ is the distance of $u$ from the set $\mathscr{S}$

$$
\operatorname{dist}(u, \mathscr{S})=\inf _{v \in \mathscr{S}}\|u-v\|
$$

Since $y_{0}$ and $y_{1}$ are both in the convex set $\mathscr{S}$, the vector $\delta y=y_{1}-y_{0}$ is in the tangent cone ${ }^{4}$. Then, for all $i$,

$$
y_{1}-y_{0}=-\frac{b_{i} c_{i}^{\top}}{c_{i}^{\top} b_{i}} y_{0} \in \mathscr{T}\left(y_{0}\right)
$$

The tangent cone to a convex set is convex, thus by taking arbitrary nonnegative numbers $\tilde{d}_{i} \geq 0$ and combining the vectors achieved for all $i$ we get, for the derivative,

$$
\dot{x}=\sum_{i=1}^{q}\left(-\tilde{d}_{i}\right) \frac{b_{i} c_{i}^{\top}}{c_{i}^{\top} b_{i}} y_{0}=\sum_{i=1}^{q} b_{i} d_{i} c_{i}^{\top} y_{0} \in \mathscr{T}\left(y_{0}\right)
$$

where $d_{i} \doteq-\tilde{d}_{i} /\left(c_{i}^{\top} b_{i}\right) \geq 0$. To conclude that $\mathscr{S}$ is positively invariant for (10), we now invoke Nagumo's theorem [30, 6], which states that any convex and compact set $\mathscr{S}$ is positively invariant for (10) if and only if $\dot{x} \in \mathscr{T}(x)$ for all $x \in \partial \mathscr{S}$.

Remark 3.1 In general the theorem holds in one direction only: the differential inclusion is stable if the difference inclusion is stable. The opposite is not true: take $q=1$ and the stable $A_{1}=\left[\begin{array}{rr}0 & 1 \\ -1 & 0\end{array}\right]$, and note that $I+d_{1} A_{1}$ is unstable.

\footnotetext{
4 we would have $\operatorname{dist}\left(y_{0}+h\left(y_{1}-y_{0}\right), \mathscr{S}\right) \equiv 0$ for $h<1$
}

We need now to remind the concept of polyhedral function. Given a full row rank matrix $X \in \mathbb{R}^{n \times s}$, the following polyhedral norm can be defined

$$
V_{X}(x)=\inf \left\{\|w\|_{1}: X w=x, w \in \mathbb{R}^{s}\right\}
$$

The vertices of the unit ball of $V_{X}(x)$ are the columns of matrix $X$ and their opposites. The dual expression is

$$
V^{F}(x)=\|F x\|_{\infty}
$$

where $F \in \mathbb{R}^{s \times n}$ has full column rank. In this case the facets of the unit ball are on the planes $F_{k} x=1$ or $F_{k} x=-1$, where $F_{k}$ is the $k$-th row of $F$.

We say that $V_{X}(x)$ is a weak Lyapunov function if it is nonincreasing along all possible system trajectories.

Theorem 3.1 admits the following corollary.

Corollary 3.1 (11) is marginally stable and has a weak polyhedral Lyapunov function if and only if (10) is marginally stable and has the same weak Lyapunov function.

Proof It is immediate since, by homogeneity, a norm is a weak Lyapunov function iff its unit ball, which is a $C$-set, is positively invariant. For the proof of Theorem 3.1, such a unit ball is positively invariant for (11) if and only if it is positively invariant for (10).

The main asymptotic stability result is summarized next.

Theorem 3.2 If (11) admits a weak Lyapunov function, then

1. (7) is stable for $\varepsilon=0$;

2. (7) is asymptotically stable for $\varepsilon>0$;

3. (1) is structurally convergent.

\subsection{Computational procedure}

Given matrix $X$, let $Y=\operatorname{mr}(X)$ be its minimal representation, namely the (unique) minimal subset of columns of $X$ for which

$$
V_{X}(x)=V_{Y}(x),
$$

achieved from $X$ by removing all the redundant vertices. Let us define the following iterate in the set of polyhedra:

$$
X^{k+1}=\Psi\left(X^{k}\right)
$$

where

$$
\Psi(X)=\operatorname{mr}\left[\begin{array}{llll}
X & \Phi_{1} X & \cdots & \Phi_{q} X
\end{array}\right]
$$

Lemma 3.2 [5] The linear differential inclusion (11) has a polyhedral Lyapunov function $V_{X}$, with $X$ full row rank matrix, if and only if $\Psi$ has a fixed point $\Psi(X)=X$, with $X$ full row rank matrix. 
For unitary networks, since $c_{i}^{\top} b_{i}=-1 \forall i$, the computation turns out to be particularly efficient, as shown next.

Proposition 3.1 Assume that $c_{i}^{\top} b_{i}=-1$ for all $i$. Then, for any $X$ integer, the sequence $X^{k}$ is integer.

This proposition is obvious from (9), because it implies that $\Phi_{k}$ are integer matrices. Its importance lies on the fact that, for the specific problem of structural stability, we can determine polyhedral functions of a special structure, namely with integer vertices. Moreover, we can define a stopping criterion for the procedure.

We can start with the computation of $X^{k}$ from any arbitrary full row rank integer matrix $X^{0}$. For simplicity, we can set $X^{0}=[-I I]$. Then the procedure works as follows ${ }^{5}$.

1. Fix $v>1$, integer. Let $X^{0}:=[-I I]$

2. Compute the sequence (12), until either

Successful stop: $X^{k}=X^{k-1}$,

Unsuccessful stop: $\max _{i j}\left|X^{k}\right|_{i j}>v$.

Proposition 3.2 If $c_{i}^{\top} b_{i}=-1$, then the previous procedure stops in a finite number of steps.

The proposition proof is trivial, because the number of integer matrices which satisfy the condition $\max _{i j}|X|_{i j} \leq v$ is finite and then the iterate $X^{k}$ can either leave the set or hit a fixed point.

To choose the value of $v$, consider that, if the procedure does not converge with a certain $v$, there will be a continuous-time trajectory originating from $\|x(0)\|_{1} \leq 1$ such that $\|x(t)\|_{\infty}$ approaches the value $v$ for large $t$, since continuous-time trajectories can get arbitrarily close to discrete-time trajectories. Since $\|x\|_{1} \geq\|x\|_{\infty}, v$ corresponds to the tolerance we accept for the ratio of the maximum 1-norm during the transient evolution of the system to the initial 1-norm, and can be fixed accordingly.

We can similarly prove the next corollary.

Corollary 3.2 If $c_{i}^{\top} b_{i}=-1$, then stability of (7) is equivalent to the existence of a polyhedral Lyapunov function.

We can consider a dual procedure having the same property. Precisely, the iteration can be applied to the dual system

$$
x_{k+1}=\Phi(k)^{\top} x_{k}
$$

The stability of the primal and the dual system are equivalent [8]. Therefore the dual procedure converges iff the primal does. In case of convergence to a matrix $\bar{X}$, the primal system admits the polyhedral Lyapunov function $V^{F}(x)=\left\|\bar{X}^{\top} x\right\|_{\infty}$.

\footnotetext{
5 Matlab code available at

http://users.dimi.uniud.it/ franco.blanchini/polychem.zip
}

We finally present an equivalence result: a polyhedral function is a Lyapunov function for the nonlinear system iff it is a Lyapunov function for the linear differential inclusion.

Theorem 3.3 Let $\bar{x}$ be a steady state of (1). Then the system is stable with a polyhedral Lyapunov function $V(x-\bar{x})$ for any possible choice of functions $g$ satisfying our assumptions if and only if $V(x-\bar{x})$ is a Lyapunov function for (7) $\forall \varepsilon \geq 0$.

Proof Just the "only if" part needs to be proved. If $V(x-\bar{x})$ is a Lyapunov function for the nonlinear system, it must be a local Lyapunov function for the linearized system $\dot{z}=J z$, where $J$ is the Jacobian. The Jacobian of the system has exactly the structure of the state matrix of the differential inclusion: $J=B D C$, where $D$ includes the partial derivatives computed in $\bar{x}$. If $g$ is arbitrary, then $D$ is arbitrary diagonal with nonnegative diagonal elements. Then the proof follows immediately.

\section{Non-unitary networks and special cases}

The procedure can be applied to non-unitary systems without conceptual restrictions. However, since integer terms of magnitude greater than 1 can appear, the procedure might not converge. In this case, it is recommended to normalize the columns of $B$ or the rows of $C$ (this is always possible, because it is equivalent to the substitution $D_{i}:=\kappa D_{i}$ ), in order to get 1 as the maximum magnitude. For example, the reactions $2 A+B \stackrel{g_{2 a b}}{\longrightarrow} C, B \stackrel{g_{b}}{\longrightarrow} \emptyset$ and $C \stackrel{g_{c}}{\longrightarrow} \emptyset$ correspond to

$$
B=\left[\begin{array}{rrcr}
-1 & -1 & 0 & 0 \\
-\frac{1}{2} & -\frac{1}{2} & -1 & 0 \\
\frac{1}{2} & \frac{1}{2} & 0 & -1
\end{array}\right], \quad C=\left[\begin{array}{llll}
1 & 0 & 0 & 0 \\
0 & 1 & 1 & 0 \\
0 & 0 & 0 & 1
\end{array}\right]^{\top}
$$

In this case the procedure converges, yet it generates a unit ball with non-integer vertices. Due to non-integer values, finite time convergence is not assured in general. Interestingly, decomposing the $g_{2 a b}$ reaction as $A+B \stackrel{g_{a b}}{\longrightarrow} D, A+D \stackrel{g_{a d}}{\longrightarrow} C$ or as $A+A \stackrel{g_{a a}}{\longrightarrow} D, B+D \stackrel{g_{b d}}{\longrightarrow} C$ still leads to convergence in both cases.

There are special structures for which there is no need to apply the procedure, since we can show they admit a polyhedral Lyapunov function. This is the case, for instance, of compartmental systems [29], which are formed by arcs as in Fig. 1 (a), (b) and (c). Actually, also arcs as in Fig. 1 (d) are admitted.

Proposition 4.1 Networks containing only reactions of the types in Fig. 1 (a), (b), (c) and (d) admit a polyhedral Lyapunov function $\|x\|_{1}$ (the proposed procedure, initialized with $[-I I]$, stops at the first step, yielding $[-I I])$.

In fact, in the case of each of these arcs, the iterate $\Psi$ maps each versor $\pm e_{h}$ in another versor, thus $\Psi$ has a fixed point 
$X=\left[\begin{array}{ll}-I & I\end{array}\right]$ and $V_{X}$ is a polyhedral Lyapunov function; linearity assures that any combination of arcs of these types admits the same polyhedral Lyapunov function.

Applying the procedure can turn out to be unnecessary also in the case of networks which are subsets/supersets of others already tested. A network $\mathscr{N}_{1}$ is a subset of $\mathscr{N}_{2}$ if it is achieved from $\mathscr{N}_{2}$ by removing arcs. In this case we say that $\mathscr{N}_{2}$ is a superset of $\mathscr{N}_{1}$.

Lemma 4.1 If a certain biochemical network admits a polyhedral Lyapunov function, then any of its subsets admits a polyhedral Lyapunov function too. Conversely, if a certain biochemical network does not admit polyhedral Lyapunov functions, then none of its supersets can admit a polyhedral Lyapunov function.

\section{Boundedness and local stability}

\subsection{Boundedness}

We have seen that, if the associated differential inclusion admits a polyhedral function, then, by Theorem 3.2 and Corollary 2.1, the original system has a bounded solution. Yet the system might be bounded even though it does not pass the "polyhedral" test. Boundedness is a fundamental property on its own [4] and we wish to investigate it further by providing a different test, inspired by the proposed procedure, but less conservative. The idea is to absorb the system in a positive differential inclusion. The trick is to divide and multiply each negative term appearing in an equation by the variable associated with that equation, for instance

$$
\dot{a}=\cdots-g(a, b) \cdots=\cdots-\frac{g(a, b)}{a} a \cdots=\cdots-k a \cdots,
$$

and to write in the same way the same positive term in the other equations. We thus achieve an absorbing differential inclusion which, if the system is bounded, is associated with a Metzler matrix.

Example 5.1 Consider the chemical system

$$
\begin{array}{ll}
A+B \frac{g_{a b}(a, b)}{} C, & \emptyset \stackrel{a_{0}}{\longrightarrow} A, \\
C+D \stackrel{g_{c d}(c, d)}{ } E, & \emptyset \stackrel{b_{0}}{\longrightarrow} B, \\
A+E \stackrel{g_{a e}(a, e)}{\square} \emptyset, & \emptyset \stackrel{d_{0}}{\longrightarrow} D,
\end{array}
$$

corresponding to the graph named Berlioz5 in Fig. 4, and the associated ODE model

$$
\begin{aligned}
& \dot{a}=-g_{a b}(a, b)-g_{a e}(a, e)+a_{0} \\
& \dot{b}=-g_{a b}(a, b)+b_{0} \\
& \dot{c}=g_{a b}(a, b)-g_{c d}(c, d) \\
& \dot{d}=-g_{c d}(c, d)+d_{0} \\
& \dot{e}=g_{c d}(c, d)-g_{a e}(a, e)
\end{aligned}
$$

If we denote $\alpha=g_{a b}(a, b) / a, \quad \beta=g_{a e}(a, e) / a, \quad \gamma=$ $g_{a b}(a, b) / b, \delta=g_{c d}(c, d) / c, \eta=g_{c d}(c, d) / d, \zeta=g_{a e}(a, e) / e$, we can rewrite the system as

$$
\left[\begin{array}{c}
\dot{a} \\
\dot{b} \\
\dot{c} \\
\dot{d} \\
\dot{e}
\end{array}\right]=\left[\begin{array}{ccccc}
-(\alpha+\beta) & 0 & 0 & 0 & 0 \\
0 & -\gamma & 0 & 0 & 0 \\
\alpha / 2 & \gamma / 2 & -\delta & 0 & 0 \\
0 & 0 & 0 & -\eta & 0 \\
0 & 0 & \delta / 2 & \eta / 2 & -\zeta
\end{array}\right]\left[\begin{array}{c}
a \\
b \\
c \\
d \\
e
\end{array}\right]+\left[\begin{array}{c}
a_{0} \\
b_{0} \\
0 \\
d_{0} \\
0
\end{array}\right] .
$$

Defining the matrices $\Phi_{k}$ as before, we can see that, for unitary systems, they are nonnegative but, unfortunately, not necessarily integer. Then we can use an iterative procedure initialized with matrix $I$. The sequence $X^{k}$ would include only nonnegative matrices, non-integer in general. The condition $X^{k}=X^{k-1}$ would tell us that the polyhedron defined as the convex hull of the columns of $X^{k}$ and 0

$$
\mathscr{P}=\left\{x=X^{k} w: \quad \sum_{i} w_{i} \leq 1\right\}
$$

is positively invariant for the discrete-time system and also for the differential inclusion, with the difference that the previous stopping criterion of Proposition 3.2 would be not valid anymore. This would imply boundedness for $g_{0}=0$ and, assuming $\varepsilon>0$, boundedness for arbitrary $g_{0}>0$ constant. Actually, it is sufficient that the additive term $g_{0}>0$ is bounded. This is relevant to systems including functions of the form $g\left(\bar{x}_{i}-x_{i}\right)$, with $0 \leq x_{i} \leq \bar{x}_{i}$, which can now be handled just as bounded terms.

Note that in most cases we do not need to use the procedure if we notice that, as in Example 5.1, the positive system is diagonally dominant. In particular, we have the following.

Proposition 5.1 A system formed only by arcs (a), (b), (c), (d), (e), ( $g),(h)$ as in Fig. 1 is structurally bounded.

Proof It is possible to verify that the matrix is at least weakly diagonally dominant in presence of $\operatorname{arcs}(\mathrm{a}),(\mathrm{b}),(\mathrm{c}),(\mathrm{d}),(\mathrm{e})$, $(\mathrm{g}),(\mathrm{h})$. In the case of diagonal dominance, the unit simplex

$$
\mathscr{P}=\left\{x: \sum_{i} x_{i} \leq 1\right\}
$$

is positively invariant for both the discrete and the continuous time systems.

Conversely, diagonal dominance is not assured in the presence of $\operatorname{arcs}(\mathrm{f})$, (i).

\subsection{Local dissipativity and mismatch}

In the previous sections we have assured asymptotic stability by introducing the $\varepsilon$ dissipativity. Yet, we have added the 
same level of dissipativity to each node and it is natural to ask whether the system tolerates a mismatch in the dissipation terms. Instead of (3) and (7), we may consider a system which has in general different dissipation terms:

$$
\dot{x}(t)=\left[-\Delta I+\sum_{i=1}^{q} b_{i} d_{i}(t) c_{i}^{\top}\right] x(t), \quad x(0)=x_{0}
$$

where $\Delta$ is a diagonal matrix with nonnegative elements, which includes $\Delta=\varepsilon I$ as a special case. We have the following.

Proposition 5.2 Assume that the system passes the computational test with a Lyapunov function $V_{X}$ (which means that $V_{X}$ is a Lyapunov function for (13) with $\Delta=0$ ). Then $V_{X}$ is a Lyapunov function for (13) if and only if it is a Lyapunov function for the $\Delta$-system

$$
\dot{x}(t)=-\Delta x(t)
$$

Proof If $V_{X}$ is a Lyapunov function for (13), since $d_{i}(t) \equiv 0$ is a possible realization, it is necessarily a Lyapunov function for the $\Delta$-system. Conversely, if $V_{X}$ is a Lyapunov function for the $\Delta$-system and, by assumption, for (13) with $\Delta=0$, by linearity it is a Lyapunov function for (13).

In the case of important mismatches in the dissipative terms, we can assume $\Delta$ to be bounded in a set of the form

$$
\mathscr{D}=\left\{\Delta: 0 \leq \Delta_{i}^{-} \leq \Delta_{i} \leq \Delta_{i}^{+}\right\}
$$

and check if the provided function works for the $\Delta$-system. This check requires linear programming [6]. Precisely, it is necessary and sufficient that, for each column $x_{k}$ of the matrix $X$ representing the function, we have

$$
\Delta x_{k} \in \mathscr{T}\left(x_{k}\right)
$$

where $\mathscr{T}\left(x_{k}\right)$ is the tangent cone whose generators are $-x_{k}+$ $x_{i}$ and $-x_{k}-x_{i}$, for all columns $x_{i}$ of $X$. In principle this is an LP problem which has to be solved for all vertices of $\mathscr{D}$ and all columns of $X$.

\subsection{Local stability within the stoichiometric compatibility class}

The $\varepsilon$ perturbation definition we have used so far may seem unnatural for reactions restricted in a stoichiometric compatibility class. If we assume $\varepsilon=0$, a polyhedral Lyapunov function may assure only marginal stability of the system. Still we may perform a test which guarantees at least local asymptotic stability of the equilibrium.

We start by stating the following.
Theorem 5.1 [8] A linear continuous-time and timeinvariant system admits polyhedral Lyapunov functions if and only if it is stable (at least marginally) and there are no purely imaginary eigenvalues (i.e. only $\lambda=0$ is admitted).

We use the theorem to show that, if we have a polyhedral Lyapunov function, then asymptotic stability is equivalent to non-singularity inside the stoichiometric compatibility class. Let $z=x-\bar{x}$ and consider the orthogonal transformation

$$
\left[\begin{array}{l}
H^{\top} \\
K^{\top}
\end{array}\right] z=\left[\begin{array}{l}
z_{H} \\
z_{K}
\end{array}\right], \quad z=\left[\begin{array}{ll}
H & K
\end{array}\right]\left[\begin{array}{l}
z_{H} \\
z_{K}
\end{array}\right],
$$

where $H$ is an orthonormal basis of $\operatorname{ker}\left[S^{\top}\right]$ (hence $H^{\top} S=0$ ) and $K$ is an orthonormal basis of $\operatorname{Ra}[S]$. Thus, if we put $g_{0}=0$ in (1), we have $H^{\top} \dot{x}=0$. By means of a state transformation, system (5) with $\varepsilon=0$ can be rewritten as

$$
\left[\begin{array}{c}
\dot{z}_{H} \\
\dot{z}_{K}
\end{array}\right]=\left[\begin{array}{c}
H^{\top} \\
K^{\top}
\end{array}\right] B D C\left[\begin{array}{ll}
H & K
\end{array}\right]\left[\begin{array}{c}
z_{H} \\
z_{K}
\end{array}\right] .
$$

Since $B$ is formed by columns of $S, H^{\top} B=0$ and

$$
\left[\begin{array}{c}
\dot{z}_{H} \\
\dot{z}_{K}
\end{array}\right]=\left[\begin{array}{cc}
0 & 0 \\
B_{K} D C_{H} & B_{K} D C_{K}
\end{array}\right]\left[\begin{array}{c}
z_{H} \\
z_{K}
\end{array}\right]
$$

where $B_{K}=K^{\top} B, C_{K}=C K$ and $C_{H}=C H$. Therefore $z_{H}(t)=z_{H}(0)$ is constant. If $\bar{x}$ is an equilibrium in the same stoichiometric class of $x(0)$, we have $z_{H}(0)=0$ and then

$$
\dot{z}_{K}=B_{K} D C_{K} z_{K}
$$

Thus we just need to assess the asymptotic stability of this latter system. If a polyhedral Lyapunov function has been found for the original system, also the $z_{K}$ subsystem admits a polyhedral Lyapunov function, because it has been obtained by a linear state transformation. Hence, in view of Theorem 5.1 , we conclude the following.

Proposition 5.3 Assume that the system admits a polyhedral Lyapunov function. Let $\bar{x}$ be an equilibrium point in the stoichiometric compatibility class of $x(0)=x_{0}$. Assume that all the partial derivatives of the functions $g_{k}$ are non-zero at the equilibrium. Then such an equilibrium is asymptotically stable iff $K^{\top} B D C K$ is structurally non-singular, where $K$ is any basis of $R a[S]$.

Note that, since we are considering a non-singularity problem, any basis $K$, not necessarily orthonormal, is suitable.

The only issue left is how to check the non-singularity of $K^{\top} B D C K=B_{K} D C_{K}$. To this aim, we propose a test which is an improved version of that proposed in [10]. Notice that

$$
\psi\left(d_{1}, d_{2}, \ldots, d_{q}\right)=\operatorname{det}\left[-B_{K} D C_{K}\right]
$$


is a multi-affine function of the nonnegative diagonal elements $d_{k}$ of $D$. To verify whether $\psi\left(d_{1}, d_{2}, \ldots, d_{q}\right) \neq 0$, since $d_{k}$ are arbitrary nonnegative scalars, we can normalize them as $0 \leq d_{k} \leq 1$. Then we consider the hypercube $\mathscr{C}_{d}=\left\{d_{k}: 0 \leq d_{k} \leq 1, k=1, \ldots, q\right\}$. Since matrices $B D C$ and $B_{K} D C_{K}$ have the same structure, we can equivalently analyze the function $\varphi\left(d_{1}, d_{2}, \ldots, d_{q}\right)=\operatorname{det}[-B D C]$ (which must be nonnegative if the system admits a polyhedral Lyapunov function). If we denote by $D^{(v)}$ the matrices corresponding to the vertices of the hypercube $\mathscr{C}_{d}$, we can prove the following.

Proposition $5.4 \operatorname{det}[-B D C]>0 \forall D>0$ if and only if $\operatorname{det}[-B C]>0$ and $\operatorname{det}\left[-B D^{(v)} C\right] \geq 0 \forall v$.

Proof Necessity obviously descends from continuity arguments. We prove sufficiency by contradiction. Assume there is an internal point $d^{*}>0$ of the hypercube such that $\varphi\left(d_{1}^{*}, d_{2}^{*}, \ldots, d_{q}^{*}\right)=0$. We recall that a multi-affine function defined on a hypercube reaches its minimum (and maximum) value on a vertex of the hypercube. Then, if we consider variations along the direction of $0 \leq d_{1} \leq 1$, we must have $\varphi\left(1, d_{2}^{*}, \ldots, d_{q}^{*}\right)=0$. If we repeat the argument for all the directions, we conclude that $\varphi(1,1, \ldots, 1)=0$, in contradiction with the assumption $\operatorname{det}[-B C]>0$.

This result allows us to assess the non-singularity of the matrix inside the hypercube $\mathscr{C}_{d}$ by simply checking the value of $\psi\left(d_{1}, d_{2}, \ldots, d_{q}\right)=\operatorname{det}\left[-K^{\top} B D C K\right]$ on the vertices of $\mathscr{C}_{d}$, thus on a finite number of points.

\section{Examples}

To evidence the potentiality of the proposed procedure, we have performed a stability test and a boundedness test for a large number of examples of biochemical networks, whose graphs are shown in Fig. 4. Each network is identified by the name of a famous musician and a number representing the order of the system.

Test results are reported in Table 1 . In column CV (convergence) we have reported the outcome (Yes/No) of the procedure described in Subsection 3.1. In the Yes case, we have reported the number of vertices and the number of facets of the unit ball of the polyhedral function, in the columns labelled as $n_{V}$ and $n_{F}$ respectively. We notice that the numbers $n_{V}$ and $n_{F}$ are surprisingly small, while in general polyhedral Lyapunov functions can be extremely complex [8]. The primal and the dual procedure may produce quite different numbers. Clearly they are always consistent with the verdict on the existence of the function. We have reported the rank of $S$ in column $\mathrm{r}(\mathrm{S})$, to evidence the dimension of the stoichiometric class, and the outcome $(\mathrm{NCC}=\mathrm{Yes} / \mathrm{No})$ of the non-singularity test in the stoichiometric class. To analyze the conservativeness of the test, we have also randomly generated points $\left(10^{5}\right)$ in the hypercube $\mathscr{C}_{d}$ and reported, in column MR, the maximum real part of the eigenvalues of
Table 1

\begin{tabular}{|c|c|c|c|c|c|c|c|}
\hline Network & $\mathrm{CV}$ & $n_{v}$ & $n_{f}$ & $\mathrm{r}(\mathrm{S})$ & $\mathrm{NCC}$ & $\mathrm{MR}$ & $\mathrm{BO}$ \\
\hline Albinoni3 & Yes & 14 & 12 & 3 & Yes & $-10^{-6}$ & Yes \\
\hline Buxtehude3 & No & - & - & 3 & No & 0.4133 & No \\
\hline Corelli3 & Yes & 6 & 6 & 3 & Yes & $-10^{-6}$ & Yes \\
\hline Frescobaldi3 & No & - & - & 3 & Yes & $-10^{-8}$ & Yes \\
\hline Pachelbel3 & No & - & - & 3 & Yes & $-10^{-6}$ & Yes \\
\hline Telemann3 & Yes & 10 & 12 & 3 & Yes & $-10^{-6}$ & Yes \\
\hline Bach4 & No & - & - & 3 & Yes & 0 & Yes \\
\hline Beethoven 4 & No & - & - & 4 & Yes & $-10^{-6}$ & Yes \\
\hline Boccherini4 & No & - & - & 4 & Yes & $-10^{-8}$ & Yes \\
\hline Čajkovskij4 & No & - & - & 4 & No & 0.2357 & Yes \\
\hline Chopin4 & Yes & 8 & 14 & 4 & Yes & $-10^{-6}$ & Yes \\
\hline Clementi4 & No & - & - & 4 & No & 0 & Yes \\
\hline Dvořák4 & No & - & - & 3 & Yes & 0 & Yes \\
\hline Fauré4 & No & - & - & 4 & No & 0 & Yes \\
\hline Gluck4 & Yes & 14 & 8 & 4 & Yes & $-10^{-7}$ & Yes \\
\hline Gounod4 & No & - & - & 4 & Yes & $-10^{-8}$ & Yes \\
\hline Händel4 & Yes & 8 & 16 & 4 & Yes & $-10^{-4}$ & Yes \\
\hline Haydn4 & Yes & 16 & 18 & 3 & Yes & 0 & Yes \\
\hline Mozart4 & Yes & 14 & 8 & 3 & Yes & 0 & Yes \\
\hline Offenbach 4 & No & - & - & 4 & Yes & $-10^{-7}$ & Yes \\
\hline Paganini4 & Yes & 14 & 18 & 4 & Yes & $-10^{-8}$ & Yes \\
\hline Pergolesi4 & No & - & - & 4 & Yes & $-10^{-8}$ & Yes \\
\hline Purcell4 & Yes & 16 & 18 & 3 & Yes & 0 & Yes \\
\hline Salieri4 & No & - & - & 4 & No & 0 & Yes \\
\hline Scarlatti4 & No & - & - & 4 & No & 0 & Yes \\
\hline Schubert4 & No & - & - & 4 & No & 0.2031 & Yes \\
\hline Schumann4 & No & - & - & 4 & No & 0 & Yes \\
\hline Vivaldi4 & No & - & - & 3 & Yes & 0 & Yes \\
\hline Berg5 & Yes & 32 & 10 & 4 & Yes & 0 & Yes \\
\hline Berlioz5 & Yes & 32 & 10 & 3 & Yes & 0 & Yes \\
\hline Brahms5 & Yes & 32 & 10 & 4 & Yes & 0 & Yes \\
\hline Elgar5 & No & - & - & 4 & Yes & 0 & Yes \\
\hline Grieg5 & Yes & 22 & 68 & 5 & Yes & $-10^{-7}$ & Yes \\
\hline Liszt5 & Yes & 28 & 66 & 5 & Yes & $-10^{-7}$ & Yes \\
\hline Martucci5 & No & - & - & 5 & Yes & $-10^{-8}$ & Yes \\
\hline Mendelssohn5 & No & - & - & 5 & No & 0 & Yes \\
\hline Rachmaninov5 & No & - & - & 4 & Yes & 0 & Yes \\
\hline Ravel5 & No & - & - & 4 & Yes & 0 & Yes \\
\hline Respighi5 & Yes & 32 & 32 & 4 & Yes & 0 & Yes \\
\hline Šostakovič5 & No & - & - & 5 & Yes & $-10^{-6}$ & Yes \\
\hline Strauss 5 & Yes & 12 & 24 & 4 & Yes & 0 & Yes \\
\hline Debussy6 & Yes & 64 & 30 & 4 & Yes & 0 & Yes \\
\hline Mahler6 & Yes & 12 & 62 & 6 & Yes & $-10^{-6}$ & Yes \\
\hline Schönberg7 & No & - & - & 7 & Yes & $-10^{-7}$ & Yes \\
\hline
\end{tabular}

$\mathbf{C V}=$ Convergence $($ Yes/No);

$n_{V}=$ number of vertices (primal procedure);

$n_{F}=$ number of facets (dual procedure);

$\mathbf{r}(\mathbf{S})=\operatorname{rank}(\mathrm{S})$;

NCC = Non-Singularity in the Stoich. Compatibility Class (Yes/No);

MR = Maximum Randomly generated eigenvalue real part;

BO $=$ Boundedness test $($ Yes/No).

all samples ${ }^{6}$. All the networks whose eigenvalues have a positive random maximum real part are recognized as unstable, as expected. However, some networks which are locally marginally stable, according to the random eigenvalues outcome, do not pass the polyhedral function test. The boundedness test is instead much less conservative, as evidenced by the results reported in the last column $(\mathrm{BO}=\mathrm{Yes} / \mathrm{No})$.

Next we show some examples of well-known models in the literature, for which we can find polyhedral functions in order to prove structural stability.

$\overline{6}$ positive values $\leq 10^{-12}$ have been considered as zero 


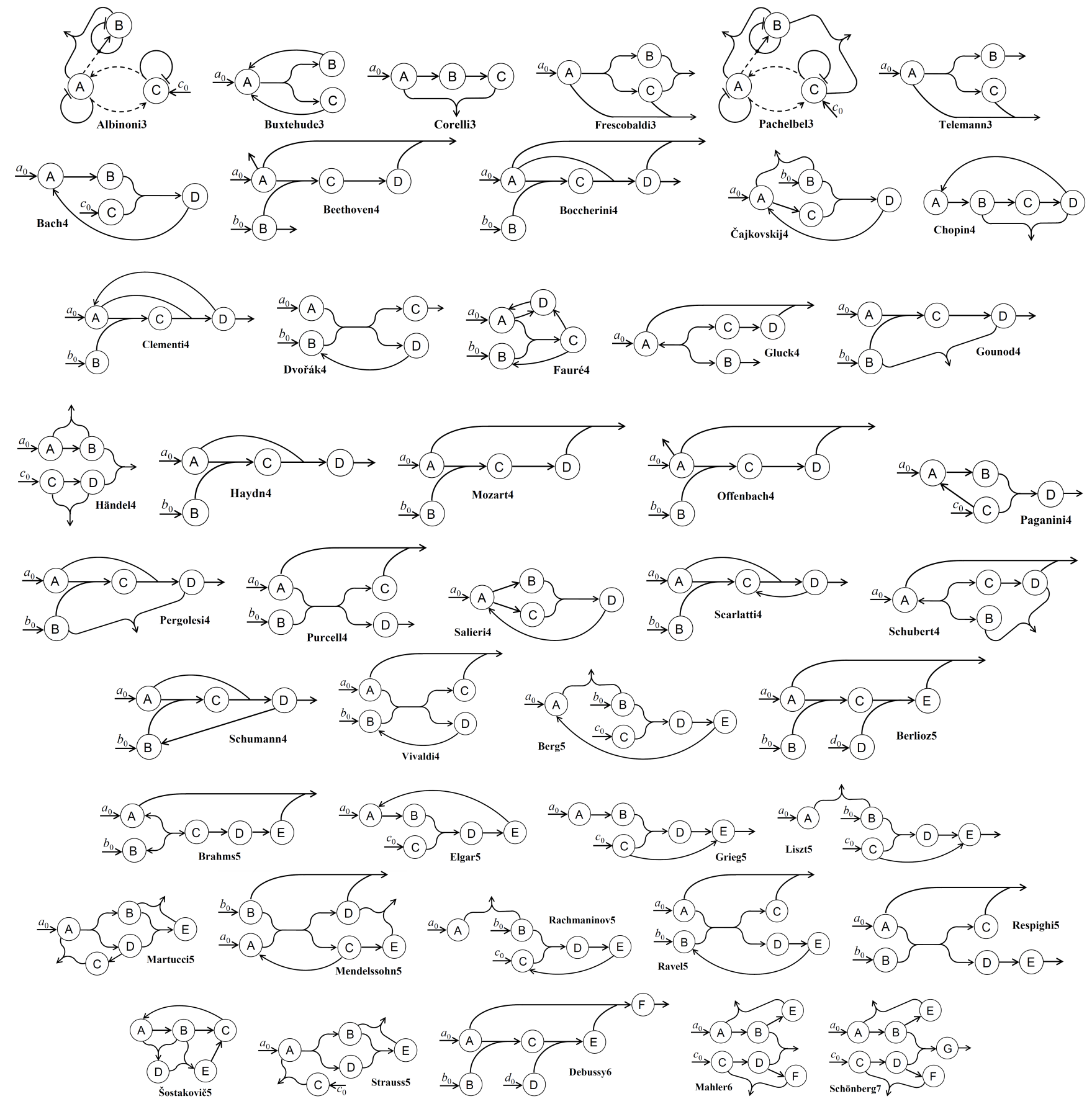

Fig. 4. Graphs of the biochemical networks tested in Section 6. Test results are reported in Table 1.

Example 6.1 Enzymatic reactions. Consider the reaction of an enzyme $E$ binding to a substrate $S$ to form a complex $C$; the product $P$ results from the modification of the substrate $S$ due to the binding with the enzyme E [1, 16, 17].

$$
\emptyset \stackrel{g_{s 0}}{\longrightarrow} S, \quad S+E \underset{g_{c}}{\stackrel{g_{e s}}{\rightleftharpoons}} C \stackrel{\bar{g}_{c}}{\longrightarrow} P+E
$$

Since $c+e=\kappa=$ const, the equations for $x=\left[\begin{array}{ll}s & e\end{array}\right]^{\top}$ are

$\dot{s}=-g_{e s}(e, s)+g_{c}(\kappa-e)+g_{s 0}$

$\dot{e}=-g_{e s}(e, s)+g_{c}(\kappa-e)+\bar{g}_{c}(\kappa-e)$

This system is bounded and, in view of Proposition 4.1, structurally stable with a Lyapunov function $\|x-\bar{x}\|_{1}$. Of course neither stability nor boundedness can be inferred for the final product $P$, which in general diverges. 
Example 6.2 A metabolic network. Consider the metabolic network proposed in [12] p. 106, with reactions

$$
\emptyset \stackrel{g_{a 0}}{\longrightarrow} A, A+C \stackrel{g_{a c}}{\longrightarrow} B+D, \quad D \stackrel{g_{d}}{\longrightarrow} C, \quad B \stackrel{g_{b}}{\longrightarrow} \emptyset
$$

If we notice that $c+d=$ const, we obtain a system in the variables $a, b$ and $c$ which turns out to be structurally stable: the procedure generates a Lyapunov function whose unit ball has 10 vertices, while the dual unit ball has 12 facets.

Example 6.3 Gene expression. Both transcription and translation can be modeled by the reduced reactions [16]

$$
A+E \underset{g_{c}}{\stackrel{g_{a e}}{\rightleftharpoons}} C, \quad C \stackrel{\bar{g}_{c}}{\longrightarrow} A+E+B .
$$

In the case of transcription, $E$ is RNA polymerase, $A$ is DNA and $B$ is produced $m R N A$. In the case of translation, $E$ stands for ribosomes, $A$ is $m R N A$ and $B$ is the produced protein. In both cases, $C$ is an intermediate complex. This mechanism, in the variables $a, c$ and $e$, is both bounded and structurally stable: the procedure generates a Lyapunov function with 12 vertices (the dual unit ball has 12 facets). Yet, if we consider the final product $B$, e.g. by including a degradation reaction $B \stackrel{g_{b}}{\longrightarrow} \emptyset$, the procedure does not converge. However, we can prove that the system is stable (hence bounded): $a, c$ and $e$, whose evolution is independent of $b$, converge to a steady state $\bar{a}, \bar{c}$ and $\bar{e}$; the equation for $b$ is $\dot{b}=\bar{g}_{c}(c)-g_{b}(b)$, therefore $b$ also converges to a steady state.

Example 6.4 MAPK pathway. Consider the open-loop MAPK pathway equations

$$
\begin{aligned}
& \dot{y}_{1}=g_{y 13}\left(\bar{y}-y_{1}-y_{3}\right)-g_{y 1}\left(x, y_{1}\right), \\
& \dot{y}_{3}=g_{x y}\left(x, \bar{y}-y_{1}-y_{3}\right)-g_{y 3}\left(y_{3}\right) \\
& \dot{z}_{1}=g_{z 13}\left(\bar{z}-z_{1}-z_{3}\right)-g_{z 1}\left(y_{3}, z_{1}\right), \\
& \dot{z}_{3}=g_{y z}\left(y_{3}, \bar{z}-z_{1}-z_{3}\right)-g_{z 3}\left(z_{3}\right),
\end{aligned}
$$

where $x$ is a constant input. The model results from the substitutions of $y_{1}+y_{2}+y_{3}=\bar{y}=$ const and $z_{1}+z_{2}+z_{3}=$ $\bar{z}=$ const in the two phosphorylation processes (see [12] p. 207 and also [22]). Numerical tests show that the system is bounded, but does not admit an overall Lyapunov function. In these cases, it is possible to adapt the framework and check only a subset of reactions. In fact, by separately analyzing the two modules of the cascade, we can see that the considered system is robustly stable. For constant $x$, the $y$ subsystem admits a polyhedral Lyapunov function (the unit ball has 6 vertices, the dual 4 facets). Hence the y variables converge to a steady state, which is asymptotically stable in view of Proposition 5.3. Convergence of $y_{3}$ to a steady state allows to apply the same analysis to the z-subsystem.

\section{Conclusions}

We have considered biochemical reaction networks with monotone reaction rates. In order to assess their structural glo- bal stability, we have devised a numerical recursive test for seeking a polyhedral Lyapunov function. In the proposed setup, the numerical procedure has shown to be very efficient in the case of unitary networks, because it operates on integer-valued matrices. The test can be conservative and may not be passed by systems which are locally stable according to a random test on the eigenvalues. In case this first test fails, a similar, less conservative procedure allows us to test whether at least the state variables are bounded. Stability and boundedness tests have been performed for many biochemical systems, including well established models in the biochemical literature. Numerical procedures have shown to be effective and useful to detect stability also in complex, quite large reaction networks: we can efficiently analyze systems up to 8-10 variables, which is still surprising in the context of polyhedral functions.

\section{References}

[1] Alon, U. (2007). Simplicity in biology. Nature, 446(7135), 497-497.

[2] Alon, U. (2006). An Introduction to Systems Biology: Design Principles of Biological Circuits. Chapman \& Hall/CRC.

[3] Anderson, D. (2008). Global asymptotic stability for a class of nonlinear chemical equations. SIAM Journal on Applied Mathematics, 68(5), 1464-1476.

[4] Angeli, D. (2011). Boundedness analysis for open chemical reaction networks with mass-action kinetics. $\mathrm{Na}$ tural Computing, 10(2), 751-774.

[5] Artstein, Z. and Rakovic, S. (2008). Feedback and invariance under uncertainty via set iterates. Automatica, 44(2), 520-525.

[6] Blanchini, F. (1991). Constrained control for uncertain linear systems. Journal of Optimization Theory and Applications, 71(3), 465-483.

[7] Blanchini, F. and Franco, E. (2012). Analysis of a class of negative feedback biochemical oscillators. In Proceedings of the American Control Conference.

[8] Blanchini, F. and Miani, S. (2008). Set-theoretic methods in control, volume 22 of Systems \& Control: Foundations \& Applications. Birkhäuser, Boston.

[9] Blanchini, F. and Franco, E. (2011). Structurally robust biological networks. Bio Med Central Systems Biology, 5(1), 74.

[10] Blanchini, F., Franco, E., and Giordano, G. (2012). Determining the structural properties of a class of biological models. In Proceedings of the IEEE Conference on Decision and Control, 5505-5510.

[11] Chaves, M. (2006). Stability of rate-controlled zerodeficiency networks. In 45th IEEE Conference on Decision and Control, 5766-5771.

[12] Chen, L., Wang, R., Li, C., and Aihara, K. (2005). Modeling Biomolecular Networks in Cells. Springer.

[13] Chesi, G. and Hung, Y. (2008). Stability analysis of uncertain genetic sum regulatory networks. Automatica, 44(9), 2298-2305.

[14] Craciun, G. and Feinberg, M. (2005). Multiple equi- 
libria in complex chemical reaction networks: I. the injectivity property. SIAM Journal on Applied Mathematics, 1526-1546.

[15] Craciun, G. and Feinberg, M. (2006). Multiple equilibria in complex chemical reaction networks: II. the species-reaction graph. SIAM Journal on Applied Mathematics, 66(4), 1321-1338.

[16] Del Vecchio, D. and Murray, R.M. (2014). Biomolecular Feedback Systems. Princeton University Press.

[17] Edelstein-Keshet, L. (2005). Mathematical Models in Biology. Society for Industrial and Applied Mathematics, Philadelphia, PA, USA.

[18] El-Samad, H., Prajna, S., Papachristodoulou, A., Doyle, J., and Khammash, M. (2006). Advanced methods and algorithms for biological networks analysis. Proceedings of the IEEE, 94(4), 832-853.

[19] Feinberg, M. (1987). Chemical reaction network structure and the stability of complex isothermal reactors I. the deficiency zero and deficiency one theorems. Chemical Engineering Science, 42, 2229-2268.

[20] Feinberg, M. (1995). The existence and uniqueness of steady states for a class of chemical reaction networks. Archive for Rational Mechanics and Analysis, 132(4), 311-370.

[21] Feinberg, M. (1995). Multiple steady states for chemical reaction networks of deficiency one. Archive for Rational Mechanics and Analysis, 132(4), 371-406.

[22] Franco, E. and Blanchini, F. (2012). Structural properties of the MAPK pathway topologies in PC12 cells. Journal of Mathematical Biology, 1-36.

[23] Hangos, K.M. (2010). Engineering model reduction and entropy-based Lyapunov functions in chemical reaction kinetics. Entropy, 12(4), 772-797.

[24] Horn, F. (1973). On a connexion between stability and graphs in chemical kinetics. I. stability and the reaction diagram. Royal Society of London Proceedings Series A, 334, 299-312.

[25] Horn, F. (1973). On a connexion between stability and graphs in chemical kinetics. II. stability and the complex graph. Royal Society of London Proceedings Series A, 334, 313-330.

[26] Horn, F. and Jackson, R. (1972). General mass action kinetics. Archive for Rational Mechanics and Analysis, 47(2), 81-116.

[27] Kim, J. and Winfree, E. (2011). Synthetic in vitro transcriptional oscillators. Molecular Systems Biology, 7, 465.

[28] Levenspiel, O. (1999). Chemical reaction engineering. John Wiley \& Sons, new York.

[29] Maeda, H., Kodama, S., and Ohta, Y. (1978). Asymptotic behavior of nonlinear compartmental systems: Nonoscillation and stability. IEEE Transactions on Circuits and Systems, 25(6), 372-378.

[30] Nagumo, M. (1942). Über die Lage der Integralkurven gewöhnlicher Differentialgleichungen. Proc. PhysMath. Soc. Japan, 24(3), 272-559.

[31] Nikolov, S., Yankulova, E., Wolkenhauer, O., and Petrov, V. (2007). Principal difference between stability and structural stability (robustness) as used in systems biology. Nonlinear Dynamics, Psychology, and Life Sciences, 11(4), 413-33.

[32] Smith, H.L. (2008). Monotone Dynamical Systems: An Introduction to the Theory of Competitive and Cooperative Systems. American Mathematical Society.

[33] Sontag, E.D. (2001). Structure and stability of certain chemical networks and applications to the kinetic proofreading model of T-Cell receptor signal transduction. IEEE Trans. Autom. Control, 46, 1028-1047. 